\title{
The Impact of Adding Sentinel Node Biopsy to Extended Pelvic Lymph Node Dissection on Biochemical Recurrence in Prostate Cancer Patients Treated with Robot-Assisted Radical Prostatectomy
}

\author{
Nikolaos Grivas ${ }^{1}$, Esther M.K. Wit ${ }^{1}$, Teele Kuusk ${ }^{1}$, Gijs H. KleinJan ${ }^{1,2}$, Maarten L. Donswijk ${ }^{3}$, Fijs W.B. van Leeuwen ${ }^{4}$, \\ and Henk G. van der Poel ${ }^{1}$ \\ ${ }^{I}$ Department of Urology, The Netherlands Cancer Institute-Antoni van Leeuwenhoek Hospital, Amsterdam, The Netherlands; \\ ${ }^{2}$ Department of Urology, Canisius Wilhelmina Ziekenhuis, Nijmegen, The Netherlands; ${ }^{3}$ Department of Nuclear Medicine, The \\ Netherlands Cancer Institute-Antoni van Leeuwenhoek Hospital, Amsterdam, The Netherlands; and ${ }^{4}$ Department of Radiology, \\ Interventional Molecular Imaging Laboratory, Leiden University Medical Center, Leiden, The Netherlands
}

The benefit of adding sentinel node biopsy (SNB) to extended pelvic lymph node dissection (ePLND) remains controversial. The aim of our study was to evaluate biochemical recurrence (BCR) after robotassisted radical prostatectomy and ePLND in prostate cancer patients, stratified by the application of SNB. The results were compared with the predictions of the updated Memorial Sloan Kettering Cancer Center nomogram. Methods: Between January 2006 and November 2016, 920 patients underwent robot-assisted radical prostatectomy and ePLND with or without SNB (184 and 736 patients, respectively). BCR was defined as 2 consecutive prostatespecific antigen rises of at least $0.2 \mathrm{ng} / \mathrm{mL}$. The Kaplan-Meier method and Cox regression analyses were used to identify predictors of BCR. Results: Median follow-up was 28 mo (interquartile range, $13-56.7 \mathrm{mo}$ ). The 5-y BCR-free survival rate was $80.5 \%$ and $69.9 \%$ in the ePLND+SNB and ePLND groups, respectively. At multivariate analysis, prostate-specific antigen level, primary Gleason grade greater than 3, seminal vesicle invasion, and higher number of removed and positive nodes were independent predictors of BCR in the ePLND group. In the ePLND+SNB group, only the number of positive nodes was an independent predictor of BCR. The overall accuracy of the Memorial Sloan Kettering Cancer Center nomogram was higher in the ePLND+SNB than in the ePLND group. However, the nomogram was underestimating the probability of BCR-free status in the ePLND+SNB group, whereas the ePLND group was performing as predicted. Conclusion: Adding SNB to ePLND improves BCR-free survival, although the precise explanation of this observation remains speculative. Our results should be interpreted cautiously, given the nonrandomized nature and the selection bias of the study.

Key Words: biochemical recurrence; pelvic lymph node dissection; prostate cancer; sentinel node; targeted lymph node biopsy

J Nucl Med 2018; 59:204-209

DOI: 10.2967/jnumed.117.195644

Received May 2, 2017; revision accepted Jun. 22, 2017.

For correspondence or reprints contact: Nikolaos Grivas, Department of Urology, The Netherlands Cancer Institute-Antoni van Leeuwenhoek Hospital, Plesmanlaan 121, 1066 CX, Amsterdam, The Netherlands.

E-mail: n.grivas@nki.nl

Published online Jul. 26, 2017.

COPYRIGHT (C 2018 by the Society of Nuclear Medicine and Molecular Imaging.
A

pproximately one quarter of prostate cancer patients undergoing radical prostatectomy develop biochemical recurrence (BCR) (1). The natural history after the occurrence of BCR is highly variable, mainly because of the heterogeneity of the disease and the differences observed on its biologic behavior. The interval until the development of clinical disease is 5-8 y, and half of these patients will die within $15 \mathrm{y}$ (2).

To assist patient counseling and treatment decision making, several nomograms have been developed and validated. These nomograms help calculate the individual risk of BCR based on pre- and postoperative clinicopathologic data (1,3-8). The recently updated postoperative Memorial Sloan Kettering Cancer Center (MSKCC) nomogram replaced the covariate of lymph node (LN) invasion by the number of positive nodes, categorized as none, 1 or 2 , and 3 or more (9). The full, updated model also includes prostatespecific antigen (PSA) level, primary and secondary pathologic Gleason grade, seminal vesicle invasion, extracapsular extension, and positive surgical margin. The additional stratification by the number of positive nodes showed favorable accuracy for predicting BCR (9).

Given the low sensitivity (49\%-66\%) of even the newest imaging methods, such as ${ }^{68} \mathrm{Ga}$-labeled prostate-specific membrane antigen PET/CT, the European Association of Urology guidelines suggest that extended pelvic LN dissection (ePLND) be considered the gold standard for LN staging of intermediate- and high-risk (>5\%) prostate cancer patients $(10,11)$. The concept of selective sentinel node (SN) biopsy (SNB) was developed mainly to avoid the toxicity of ePLND and to improve detection rates for positive SNs. SNB is a standard procedure in melanoma, breast, and penile cancer and is increasingly applied also in prostate cancer (12-14). Although SNB is still considered experimental, a recent systematic review has shown a diagnostic accuracy comparable to ePLND (15). Moreover, the combination of ePLND and SNB can increase the detection rate of affected nodes (15). Nevertheless, one open question is the ability of the procedure to increase the removal of metastatic LNs, which could result in better oncologic outcomes than ePLND-only dissection.

The aim of this study was to evaluate the BCR outcomes after robot-assisted radical prostatectomy (RARP) in prostate cancer 
patients, stratified by the additional application of SNB. The observed results were validated with the predictions of the updated MSKCC nomogram.

\section{MATERIALS AND METHODS}

\section{Study Population}

After obtaining institutional approval, we retrospectively reviewed the data of 920 consecutive prostate cancer patients who underwent RARP along with pelvic lymphadenectomy for clinically organconfined prostate cancer from January 2006 until November 2016. In 736 patients (80\%), ePLND alone was applied. In 184 patients (20\%), ePLND was combined with additional SNB (ePLND+SNB) within the scope of a clinical study. The study protocol was approved by the Institutional Committee on Ethics (Dutch trial register NL41285.031.12), and all patients gave written informed consent. Patients who declined participation were offered only ePLND. Patients who received salvage prostatectomy or adjuvant treatment or who had missing data were excluded. The indication for LN dissection was based on the guidelines of the European Association of Urology.

The primary tumor was staged by digital rectal examination, transrectal ultrasound, or MRI and classified per the 2009 TNM staging system. PSA was measured using standard assays. All patients underwent transrectal ultrasound-guided prostate biopsy, and the total number of cores, the number and percentage of positive cores, the primary and secondary Gleason grades, the number of removed and positive LNs, and the presence of seminal vesicle invasion, extracapsular extension, or a positive surgical margin were prospectively recorded. The number of positive nodes was categorized in accordance with the updated MSKCC nomogram (9). Follow-up after RARP consisted of serum PSA analyses every 4 mo for the first $3 \mathrm{y}$ and every 6 mo thereafter.

\section{SN and ePLND Technique}

The RARP, ePLND, SNB technique, and pathologic examination were performed as described earlier (16). In brief, surgery was performed by a urologist experienced in robot-assisted surgery using the da Vinci S(i) Surgical System (Intuitive Surgical Inc.). The ePLND included the removal of the nodes along the external and internal iliac artery and vein, the obturator nodes, and the nodes overlying the common iliac vessels up to the ureteral crossing. All SNs were identified using the hybrid tracer indocyanine green- ${ }^{99 \mathrm{~m}} \mathrm{Tc}$-nanocolloid (dose, $240 \mathrm{MBq}$; median particle size, $14 \mathrm{~nm}$ ), which was transrectally injected on the morning of surgery into the peripheral zone of the prostate under ultrasound guidance. Planar imaging of the pelvic area from an anterior and lateral position was performed at 15-30 min and $2 \mathrm{~h}$ after injection followed by SPECT and low-dose CT to generate a roadmap for intraoperative localization of the individual SNs (16). Surgery was planned to start from $4 \mathrm{~h}$ after injection. The SNs were cut into 2-mm sections, and hematoxylin-eosin staining was applied. A CAM5.2 antibody was used for immunohistochemical analysis (catalog no. 345779; Becton Dickinson Biosciences).

\section{Outcome Assessment}

BCR was defined as 2 consecutive PSA rises of at least $0.2 \mathrm{ng} / \mathrm{mL}$ (11). Two groups of patients were compared; that is, patients who underwent RARP and ePLND and those who underwent RARP and ePLND+SNB. The observed BCR results were validated with the predictions of the updated MSKCC nomogram. Finally, we did an additional subgroup analysis on a cohort of patients with at least 14 LNs removed.

\section{Statistical Analysis}

The Mann-Whitney nonparametric U test was used to compare the median values of baseline characteristics. The Kaplan-Meier plot was

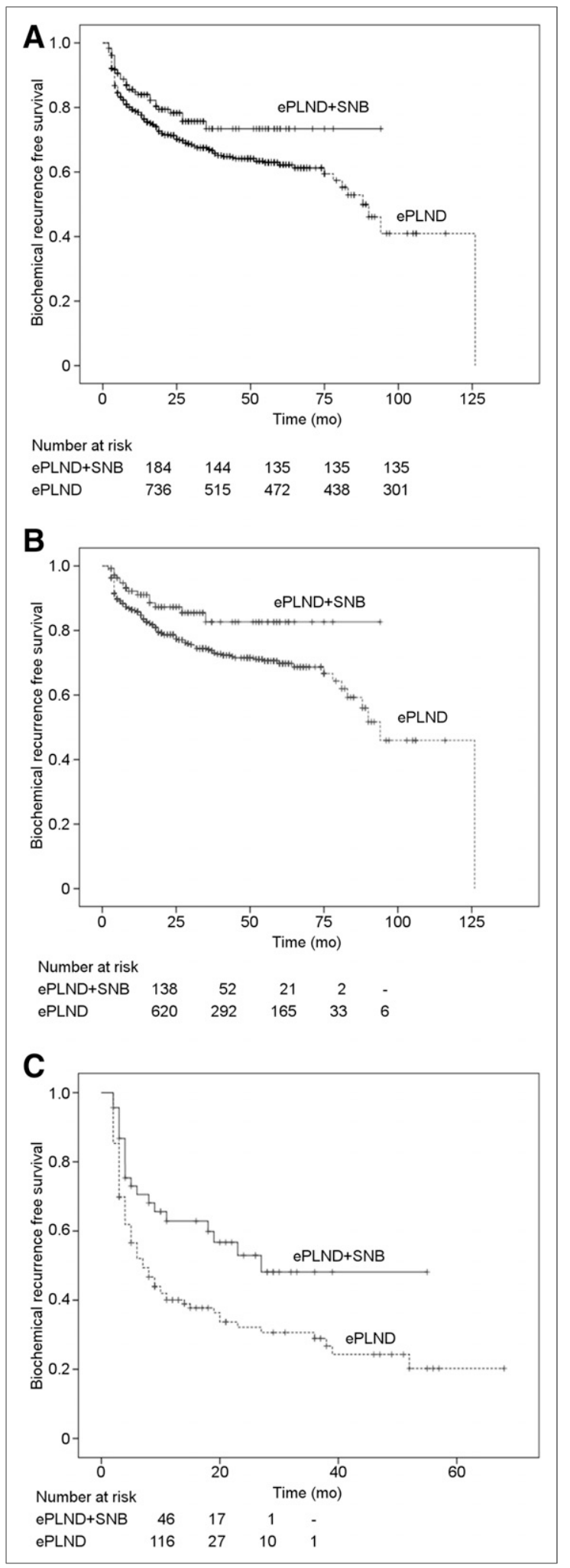

FIGURE 1. Kaplan-Meier survival curves of BCR-free survival, stratified by performance of SNB, in overall cohort (log-rank test, $P=0.03$ ) (A), in node-negative patients (log-rank test, $P=0.016)(B)$, and in node-positive patients (log-rank test, $P=0.011)(\mathrm{C})$. 
used to present BCR-free survival data, the log rank assay was used to test significance, and the Cox multivariate regression analysis was used to compare factors associated with BCR. Covariates included preoperative PSA, clinical stage, Gleason grade at primary and secondary biopsy, extracapsular extension, positive surgical margin, seminal vesicle invasion, number of removed nodes, number of positive nodes ( 0 vs. $1-2$ or $\geq 3$ ), and removal or not of SNs. The area under the curve (AUC), as well as calibration plots, were used to compare actual outcome and nomogram prediction between the 2 groups. Decision curve analyses were performed to examine the relationship between the threshold probability of BCR and the relative value of false-positive and -negative results. $P$ values of less than 0.05 were considered statistically significant. Version 22.0 SPSS software (SPSS Inc.) and the R statistical package (R Foundation for Statistical Computing) were used to perform the statistical analyses.

\section{RESULTS}

\section{Baseline Characteristics}

Baseline characteristics are presented in Supplemental Table 1 (supplemental materials are available at http://jnm.snmjournals.org). Median follow-up was 28 mo (interquartile range, 13-56.7 mo). The median number of removed LNs and the percentage of nodepositive patients were significantly higher in the ePLND + SNB group (14 vs. $9, P<0.001$, and $25 \%$ vs. $15.8 \%, P=0.003$ ), whereas median preoperative PSA was significantly higher in the ePLND group ( 9.3 vs. $8.3, P=0.004)$. Stratified by the number of positive nodes, 1 or 2 and more than 3 positive nodes were significantly more common in the ePLND+SNB group than in the ePLND group ( $17.9 \%$ vs. $11.8 \%$ and $7.1 \%$ vs. $3.9 \%[P=0.003]$ of the patients, respectively).

\section{Survival Analysis}

The 5-y BCR-free survival rate was significantly higher $(P=$ $0.03)$ in the ePLND+SNB group than in the ePLND group ( $80.5 \%$ vs. $69.9 \%$, respectively) (Fig. 1A). When patients were stratified according to $\mathrm{LN}$ stage, BCR-free survival rates were again in favor of the ePLND+SNB group (both $P \leq 0.016$; Figs. $1 \mathrm{~B}$ and $1 \mathrm{C})$.

\section{Subgroup Analysis}

In the subgroup analysis of patients with at least $14 \mathrm{LNs}$ removed, the 5-y BCR-free survival rate remained significantly higher in the ePLND+SNB group than in the ePLND group (82.7\% vs. $63.9 \%$, respectively; $P=0.001$ ). Similar results were observed when patients were stratified according to $\mathrm{LN}$ stage (node-negative: $91.3 \%$ vs. $76.2 \%, P=0.026$; node-positive: $62.1 \%$ vs. $24.1 \%$, $P=0.001)$.

\section{Univariate and Multivariate Cox Regression Models Predicting BCR}

The univariate and multivariate analyses for the entire cohort are shown in Table 1. In the univariate analysis, higher preoperative PSA (odds ratio [OR], 1.014; 95\% confidence interval [CI], 1.010-1017; $P<0.001)$, advanced clinical stage $(P<0.001)$, primary pathologic Gleason grade of more than 3 (OR, 3.746; 95\% CI, 2.923-4.801; $P<0.001$ ), positive surgical margin (OR, 2.111; 95\% CI, 1.657-2.688; $P<0.001$ ), extracapsular extension (OR, 3.314; 95\% CI, 2.566-4.280; $P<0.001$ ), higher number of removed nodes (OR, 1.024; 95\% CI, 1.004-1.044; $P=0.017)$, and higher number of positive nodes $(P<0.001)$ were predictors of BCR, whereas SNB was negatively correlated with BCR (OR, 0.682; 95\% CI, 0.479-0.969; $P=0.033$ ). The multivariate analysis showed the independent predictors of BCR to be higher PSA (OR, 1.013; 95\% CI, 1.008-1.018; $P<0.001$ ), primary pathologic Gleason grade of more than 3 (OR, 2.352; 95\% CI, 1.793-3.085; $P<0.001$ ), positive surgical margin (OR, 1.361; 95\% CI, 1.048-1.768; $P=0.021$ ), seminal vesicle invasion (OR, 1.744; 95\% CI, 1.263-2.408; $P=0.001)$, higher number of removed nodes (OR, 1.025; 95\% CI, 1.002-1.048; $P=0.032$ ), and higher number of positive nodes $(P<0.001)$, whereas SNB remained inversely correlated with $\mathrm{BCR}(\mathrm{OR}, 0.535$; 95\% CI, $0.368-0.777 ; P=0.001)$.

TABLE 1

Univariate and Multivariate Pre- and Postoperative Factors for Biochemical Recurrence in Overall Cohort

\begin{tabular}{|c|c|c|c|c|c|c|}
\hline \multirow[b]{2}{*}{ Variable } & \multicolumn{3}{|c|}{ Univariate } & \multicolumn{3}{|c|}{ Multivariate } \\
\hline & Odds & $95 \% \mathrm{Cl}$ & $P$ & Odds & $95 \% \mathrm{Cl}$ & $P$ \\
\hline Preoperative serum PSA & 1.014 & $1.010-1.017$ & 0.000 & 1.013 & $1.008-1.018$ & 0.000 \\
\hline Clinical stage & - & - & 0.000 & - & - & 0.559 \\
\hline T2 vs. T1 & 1.07 & $0.731-1.567$ & 0.728 & 0.859 & $0.583-1.266$ & 0.443 \\
\hline T3 vs. T1 & 2.359 & $1.607-3.463$ & 0.000 & 0.983 & $0.651-1.486$ & 0.937 \\
\hline Primary pathologic Gleason grade $>3$ & 3.746 & $2.923-4.801$ & 0.000 & 2.352 & $1.793-3.085$ & 0.000 \\
\hline Secondary pathologic Gleason grade $>3$ & 0.859 & $0.67-1.103$ & 0.234 & - & - & - \\
\hline SN biopsy & 0.682 & $0.479-0.969$ & 0.033 & 0.535 & $0.368-0.777$ & 0.001 \\
\hline Positive surgical margin & 2.111 & $1.657-2.688$ & 0.000 & 1.361 & $1.048-1.768$ & 0.021 \\
\hline Extracapsular extension & 3.314 & $2.566-4.280$ & 0.000 & 1.302 & $0.921-1.841$ & 0.135 \\
\hline Seminal vesicle invasion & 4.097 & $3.213-5.224$ & 0.000 & 1.744 & $1.263-2.408$ & 0.001 \\
\hline Number of removed nodes & 1.024 & $1.004-1.044$ & 0.017 & 1.025 & $1.002-1.048$ & 0.032 \\
\hline Number of positive nodes & - & - & 0.000 & - & - & 0.000 \\
\hline $1-2$ vs. 0 positive nodes & 3.783 & $2.843-5.034$ & 0.000 & 2.266 & $1.654-3.106$ & 0.000 \\
\hline$>2$ vs. 0 positive nodes & 8.447 & $5.713-12.489$ & 0.000 & 3.555 & $2.269-5.570$ & 0.000 \\
\hline
\end{tabular}


TABLE 2

Univariate and Multivariate Pre- and Postoperative Factors for Biochemical Recurrence in ePLND Group

\begin{tabular}{|c|c|c|c|c|c|c|}
\hline \multirow[b]{2}{*}{ Variable } & \multicolumn{3}{|c|}{ Univariate } & \multicolumn{3}{|c|}{ Multivariate } \\
\hline & Odds & $95 \% \mathrm{Cl}$ & $P$ & Odds & $95 \% \mathrm{Cl}$ & $P$ \\
\hline Preoperative serum PSA & 1.013 & $1.009-1.017$ & 0.000 & 1.013 & $1.008-1.018$ & 0.000 \\
\hline Clinical stage & - & - & 0.000 & - & - & 0.741 \\
\hline T2 vs. T1 & 1.129 & $0.753-1.694$ & 0.557 & 0.85 & $0.562-1.285$ & 0.440 \\
\hline T3 vs. T1 & 2.506 & $1.659-3.785$ & 0.000 & 0.884 & $0.562-1.391$ & 0.595 \\
\hline Primary pathologic Gleason grade $>3$ & 3.843 & $2.943-5.019$ & 0.000 & 2.51 & $1.875-3.36$ & 0.000 \\
\hline Secondary pathologic Gleason grade $>3$ & 0.817 & $0.627-1.066$ & 0.136 & - & - & - \\
\hline Positive surgical margin & 1.999 & $1.541-2.592$ & 0.000 & 1.304 & $0.981-1.732$ & 0.067 \\
\hline Extracapsular extension & 3.186 & $2.417-4.199$ & 0.000 & 1.289 & $0.887-1.874$ & 0.183 \\
\hline Seminal vesicle invasion & 3.850 & $2.966-4.999$ & 0.000 & 1.772 & $1.255-2.503$ & 0.001 \\
\hline Number of removed nodes & 1.043 & $1.021-1.067$ & 0.000 & 1.034 & $1.009-1.059$ & 0.007 \\
\hline Number of positive nodes & - & - & 0.000 & - & - & 0.000 \\
\hline $1-2$ vs. 0 positive nodes & 3.993 & $2.914-5.471$ & 0.000 & 2.237 & $1.578-3.173$ & 0.000 \\
\hline$>2$ vs. 0 positive nodes & 11.346 & $7.334-17.552$ & 0.000 & 3.746 & $2.257-6.217$ & 0.000 \\
\hline
\end{tabular}

The univariate analysis in the ePLND group showed the same predictors of BCR as for the entire cohort (Table 2). The results of multivariate analysis were also similar except for positive surgical margin, which was not identified as an independent predictor of BCR in this group. In the ePLND+SNB group, the predictors in univariate analysis were again the same as in the overall cohort, except for the number of removed nodes. However, in the multivariate analysis, only a higher number of positive nodes remained an independent predictor of BCR (Table 3).

\section{Observed Outcome Versus Nomogram Prediction}

On the basis of the AUC, the performance of the MSKCC nomogram was better in the ePLND+SNB group than in the ePLND group (respectively: AUC, 0.804; 95\% CI, 0.725-0.884; and AUC, 0.789; 95\% CI, 0.753-0.826). In the calibration plots (Figs. 2A and 2B), the nomogram was underestimating the probability of BCR-free status in the ePLND + SNB group (mean absolute error, 0.021) whereas the ePLND group was performing almost as predicted (mean absolute error, 0.009). The decision curve analyses indicated that the MSKCC nomogram resulted in higher net benefit in the ePLND+SNB group than in the ePLND group, for most of the examined BCR probabilities (Figs. 3A and 3B).

\section{DISCUSSION}

To the best of our knowledge, our study was the first to explore the BCR outcome of men treated by RARP and ePLND compared with a cohort of men treated with ePLND+SNB. Our results show

TABLE 3

Univariate and Multivariate Pre- and Postoperative Factors for Biochemical Recurrence in ePLND+SNB Group

\begin{tabular}{|c|c|c|c|c|c|c|}
\hline \multirow[b]{2}{*}{ Variable } & \multicolumn{3}{|c|}{ Univariate } & \multicolumn{3}{|c|}{ Multivariate } \\
\hline & Odds & $95 \% \mathrm{Cl}$ & $P$ & Odds & $95 \% \mathrm{Cl}$ & $P$ \\
\hline Preoperative serum PSA & 1.038 & $1.018-1.059$ & 0.000 & 1.013 & $0.989-1.039$ & 0.290 \\
\hline Clinical stage & - & - & 0.021 & - & - & 0.171 \\
\hline T2 vs. T1 & 0.800 & $0.261-2.454$ & 0.696 & 0.785 & $0.223-2.761$ & 0.706 \\
\hline T3 vs. T1 & 2.113 & $0.718-6.216$ & 0.174 & 1.584 & $0.469-5.345$ & 0.459 \\
\hline Primary pathologic Gleason grade $>3$ & 3.598 & $1.822-7.106$ & 0.000 & 1.622 & $0.729-3.610$ & 0.236 \\
\hline Secondary pathologic Gleason grade $>3$ & 1.430 & $0.651-3.140$ & 0.372 & - & - & - \\
\hline Positive surgical margin & 2.731 & $1.419-5.254$ & 0.003 & 1.703 & $0.852-3.405$ & 0.132 \\
\hline Extracapsular extension & 3.918 & $1.983-7.741$ & 0.000 & 1.240 & $0.475-3.235$ & 0.660 \\
\hline Seminal vesicle invasion & 6.025 & $3.094-11.731$ & 0.000 & 2.159 & $0.788-5.917$ & 0.134 \\
\hline Number of removed nodes & 0.992 & $0.941-1.045$ & 0.750 & - & - & - \\
\hline Number of positive nodes & - & - & 0.000 & - & - & 0.025 \\
\hline $1-2$ vs. 0 positive nodes & 4.218 & $2.055-8.661$ & 0.000 & 2.521 & $1.155-5.505$ & 0.020 \\
\hline$>2$ vs. 0 positive nodes & 6.060 & $2.358-15.572$ & 0.000 & 3.319 & $1.094-10.065$ & 0.034 \\
\hline
\end{tabular}



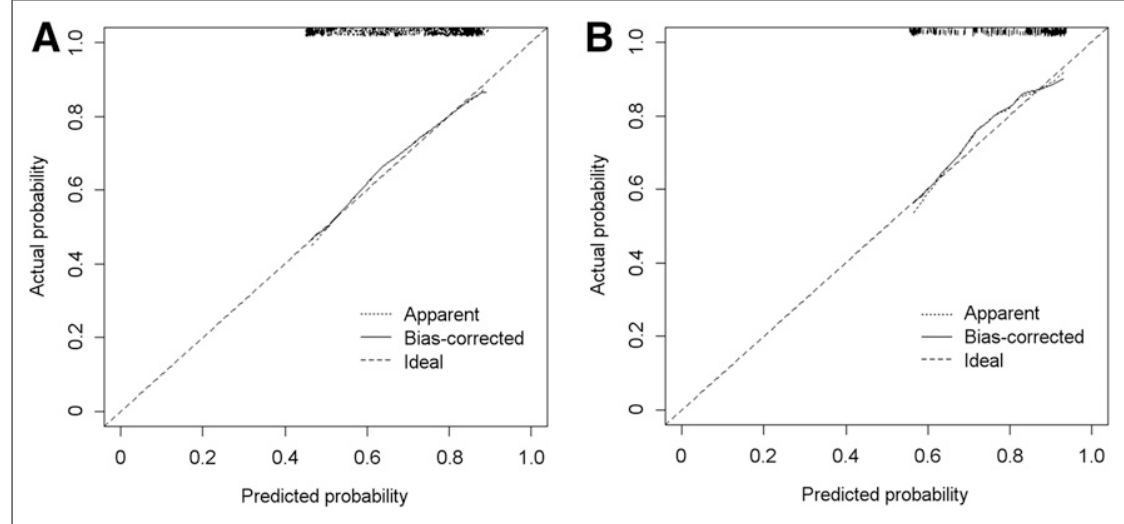

FIGURE 2. Nomogram calibration plots for ePLND group (A) and for ePLND+SNB group (B). Dotted plot indicates location of ideal nomogram, in which actual and predicted probabilities are identical. Expected performance on future data is represented through solid line. prediction was better in the ePLND + SNB group (AUC, 0.804) than in the ePLND group (AUC, 0.789). The favorable outcome observed in the calibration analysis of the ePLND+SNB group strengthens our observation of the improved $\mathrm{BCR}$-free outcome when SNB is performed. It also suggests that the improved outcome of men after SNB is independent of clinical characteristics such as Gleason grade, PSA level, and tumor stage. The decision curve analyses indicated that the net benefit of the MSKCC nomogram was higher in the ePLND + SNB group than in the ePLND group in almost the entire range of threshold probabilities.

The explanation for the improved BCRfree survival in the ePLND+SNB group compared with the ePLND group is a matter of speculation. Fossati et al., in their that when SNB was applied, there was a $17 \%$ increase in the number of patients who achieved a 5-y BCR-free status. This improved outcome was independent of LN status. In the subgroup of patients from whom more than 14 LNs were removed, a $29.4 \%$ increase in the percentage of 5-y BCR-free patients was observed compared with the ePLND-only patients. The decision to perform a subgroup analysis for men with at least $14 \mathrm{LNs}$ removed was based on previous reports demonstrating favorable survival outcomes in patients treated with ePLND and more than 14 nodes removed (17-19).

To increase the power of our analysis, we compared the observed BCR outcomes with the predictions of the recently updated postoperative MSKCC nomogram (9), performing in that way the first external validation of this nomogram. Our study demonstrated a reasonably accurate predictive accuracy of the nomogram for the entire cohort (AUC, 0.789; 95\% CI, 0.754-0.824). Moreover, the recent systematic review, reported that the therapeutic role of ePLND itself is still not evident from the current literature (20). We observed a higher number of removed and histologically positive nodes when SNB was performed. In line with these findings, a diagnostic and therapeutic effect of combining ePLND and SNB has been suggested in a systematic review from Wit et al. (15). This review indicates that the SNs were the only metastatic site in $73 \%$ of LN-positive patients whereas in 1 of 20 patients who underwent ePLND, metastatic LNs would have been missed without SNB. Winter et al. also detected more $\mathrm{LN}$-positive patients than predicted by the Briganti nomogram when sentinel lymphadenectomy was performed (21), and their SN-based nomogram demonstrated a high predictive accuracy (22). Recently, the detection rate of SNs has also shown further improvement with the application of new detection techniques, such as by combining
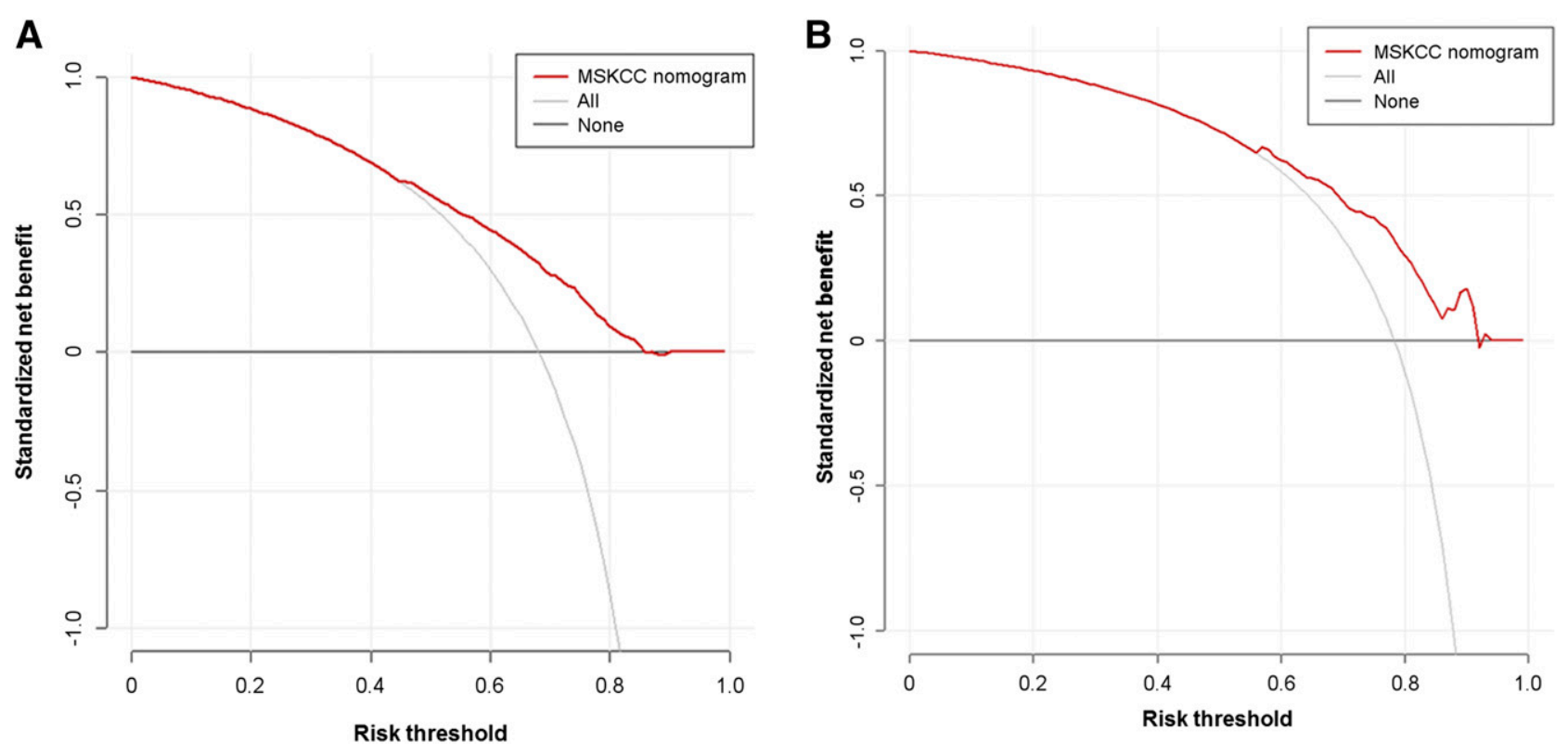

FIGURE 3. Decision curve analyses for biochemical recurrence predictions in ePLND group (A) and in ePLND+SNB group (B). Red line indicates net benefit of using updated MSKCC nomogram. Gray line indicates assumption that all patients will experience recurrence. Black line indicates assumption that no patients will experience recurrence. 
indocyanine green- ${ }^{99 m}$ Tc-nanocolloid with fluorescein (23). A recent SN consensus panel suggested that SNB might identify metastatic nodes outside the extended lymphadenectomy template but that SNB should be combined with ePLND, especially in intermediate- and high-risk patients, since positive non-SNs were often found besides the SNs $(24,25)$. In addition to the improved detection rate, targeted $\mathrm{SN}$ dissection allows for a separate and thus more accurate histopathologic examination, thereby increasing detection of (especially) small LN metastases (26). Muck et al. have shown improved clinical outcomes in patients treated with radical prostatectomy combined with ePLND+SNB when they have a low nodal tumor burden (micrometastases) (27).

In accordance with other studies (28), our Cox regression analysis showed that adverse pathologic characteristics such as a primary pathologic Gleason grade of more than 3, a positive surgical margin, seminal vesicle invasion, and extracapsular extension were independent predictors of BCR. Fischer et al. (29), in a sample of 459 men, demonstrated a 2-y BCR risk of at least 50\% in patients with a positive surgical margin, extracapsular extension, or seminal vesicle invasion. In our Cox analysis, the number of positive nodes was also an independent predictor of BCR in the overall cohort and in both groups. Moreover, the initial Stephenson nomogram (6) performed worse than the updated nomogram (AUC, 0.789 vs. 0.681 in the overall cohort), confirming the value of replacing the positive LN status in the initial nomogram with the number of positive nodes in the updated nomogram (9).

Limitations of our study include the retrospective setting, the relatively short median follow-up, and the selection bias due to a single-institution case series. In addition, the data depend on factors such as the extent of LN dissection and complexities regarding tissue handling and the accuracy of histologic detection and reporting of nodal metastases. Finally, we lacked any data on the location of the SNs and the overall survival of the patients.

\section{CONCLUSION}

Adding SNB to ePLND resulted in improved BCR-free outcome compared with use of only ePLND in men after RARP. This observation was strengthened by the improved prediction outcome as compared with the MSKCC nomogram. SNB also resulted in favorable nodal staging through the detection of more positive nodes than ePLND. SNB remains an attractive and promising staging intervention that may expand surgical options in managing men with localized prostate cancer. Further research and randomized studies to assess the effect of SNB on LN detection rate and the relationship between SNB and BCR are warranted.

\section{DISCLOSURE}

No potential conflict of interest relevant to this article was reported.

\section{REFERENCES}

1. Stephenson AJ, Scardino PT, Eastham JA, et al. Preoperative nomogram predicting the 10-year probability of prostate cancer recurrence after radical prostatectomy. J Natl Cancer Inst. 2006;98:715-717.

2. Freedland SJ, Humphreys EB, Mangold LA, et al. Risk of prostate cancerspecific mortality following biochemical recurrence after radical prostatectomy. JAMA. 2005;294:433-439.

3. Zhang YD, Wu CJ, Bao ML, et al. MR-based prognostic nomogram for prostate cancer after radical prostatectomy. J Magn Reson Imaging. 2017;45:586-596.

4. D'Amico AV, Whittington R, Malkowicz SB, et al. Biochemical outcome after radical prostatectomy, external beam radiation therapy, or interstitial radiation therapy for clinically localized prostate cancer. JAMA. 1998;280: 969-974.
5. Cooperberg MR, Pasta DJ, Elkin EP, et al. The University of California, San Francisco Cancer of the Prostate Risk Assessment score: a straightforward and reliable preoperative predictor of disease recurrence after radical prostatectomy. J Urol. 2005;173:1938-1942.

6. Stephenson AJ, Scardino PT, Eastham JA, et al. Postoperative nomogram predicting the 10-year probability of prostate cancer recurrence after radical prostatectomy. J Clin Oncol. 2005;23:7005-7012.

7. Lughezzani G, Budäus L, Isbarn H, et al. Head-to-head comparison of the three most commonly used preoperative models for prediction of biochemical recurrence after radical prostatectomy. Eur Urol. 2010;57:562-568.

8. Walz J, Chun FK, Klein EA, et al. Nomogram predicting the probability of early recurrence after radical prostatectomy for prostate cancer. J Urol. 2009;181:601-607.

9. Nguyen DP, Kent M, Vilaseca A, et al. Updated postoperative nomogram incorporating the number of positive lymph nodes to predict disease recurrence following radical prostatectomy. Prostate Cancer Prostatic Dis. 2017;20:105-109.

10. Budäus L, Leyh-Bannurah SR, Salomon G, et al. Initial experience of ${ }^{68} \mathrm{Ga}-$ PSMA PET/CT imaging in high-risk prostate cancer patients prior to radical prostatectomy. Eur Urol. 2016;69:393-396.

11. Mottet N, Bellmunt J, Bolla M, et al. EAU-ESTRO-SIOG guidelines on prostate cancer. Part 1: screening, diagnosis, and local treatment with curative intent. Eur Urol. 2017;71:618-629.

12. Rouzier R, Uzan C, Rousseau A, et al. Multicenter prospective evaluation of the reliability of the combined use of two models to predict non-sentinel lymph node status in breast cancer patients with metastatic sentinel lymph nodes: the MSKCC nomogram and the Tenon score: results of the NOTEGS study. $\mathrm{Br} \mathrm{J}$ Cancer. 2017;116:1135-1140.

13. Schubert T, Uphoff J, Henke RP, Wawroschek F, Winter A. Reliability of radioisotope-guided sentinel lymph node biopsy in penile cancer: verification in consideration of the European guidelines. BMC Urol. 2015;15:98.

14. van der Poel HG, Meershoek P, Grivas N, KleinJan G, van Leeuwen FW, Horenblas S. Sentinel node biopsy and lymphatic mapping in penile and prostate cancer. Urologe A. 2017;56:13-17.

15. Wit EM, Acar C, Grivas N, et al. Sentinel node procedure in prostate cancer: a systematic review to assess diagnostic accuracy. Eur Urol. 2017;71:596-605.

16. KleinJan GH, van den Berg NS, Brouwer OR, et al. Optimisation of fluorescence guidance during robot-assisted laparoscopic sentinel node biopsy for prostate cancer. Eur Urol. 2014;66:991-998.

17. Abdollah F, Gandaglia G, Suardi N, et al. More extensive pelvic lymph node dissection improves survival in patients with node-positive prostate cancer. Eur Urol. 2015;67:212-219.

18. Briganti A, Blute ML, Eastham JH, et al. Pelvic lymph node dissection in prostate cancer. Eur Urol. 2009;55:1251-1265.

19. Kluth LA, Abdollah F, Xylinas E, et al. Clinical nodal staging scores for prostate cancer: a proposal for preoperative risk assessment. Br J Cancer. 2014;111:213-219.

20. Fossati N, Willemse PM, Van den Broeck T, et al. The benefits and harms of different extents of lymph node dissection during radical prostatectomy for prostate cancer: a systematic review. Eur Urol. 2017;72:84-109.

21. Winter A, Kneib T, Henke RP, Wawroschek F. Sentinel lymph node dissection in more than 1200 prostate cancer cases: rate and prediction of lymph node involvement depending on preoperative tumor characteristics. Int J Urol. 2014;21:58-63.

22. Winter A, Kneib T, Rohde M, Henke RP, Wawroschek F. First nomogram predicting the probability of lymph node involvement in prostate cancer patients undergoing radioisotope guided sentinel lymph node dissection. Urol Int. 2015;95:422-428.

23. van den Berg NS, Buckle T, KleinJan GH, van der Poel HG, van Leeuwen FW. Multispectral fluorescence imaging during robot-assisted laparoscopic sentinel node biopsy: a first step towards a fluorescence-based anatomic roadmap. Eur Urol. 2017;72:110-117.

24. van der Poel HG, Wit EM, Acar C, et al. Sentinel node biopsy for prostate cancer: report from a consensus panel meeting. BJU Int. 2017;120:204-211.

25. Holl G, Dorn R, Wengenmair H, Weckermann D, Sciuk J. Validation of sentinel lymph node dissection in prostate cancer: experience in more than 2,000 patients. Eur J Nucl Med Mol Imaging. 2009;36:1377-1382.

26. Acar C, Kleinjan GH, van den Berg NS, Wit EM, van Leeuwen FW, van der Poel HG. Advances in sentinel node dissection in prostate cancer from a technical perspective. Int J Urol. 2015;22:898-909.

27. Muck A, Langesberg C, Mugler M, Rahnenführer J, Wullich B, Schafhauser W. Clinical outcome of patients with lymph node-positive prostate cancer following radical prostatectomy and extended sentinel lymph node dissection. Urol Int. 2015;94:296-306.

28. Karl A, Buchner A, Tympner C, et al. Risk and timing of biochemical recurrence in pT3aN0/Nx prostate cancer with positive surgical margin: a multicenter study. Radiother Oncol. 2015;116:119-124.

29. Fischer S, Lin D, Simon RM, et al. Do all men with pathological Gleason score 8-10 prostate cancer have poor outcomes? Results from the SEARCH database. BJU Int. 2016;118:250-257. 